\title{
Association between Resting-State Coactivation in the Parieto-Frontal Network and Intelligence during Late Childhood and Adolescence
}

\author{
C. Li and L. Tian
}

\begin{abstract}
BACKGROUND AND PURPOSE: A number of studies have associated the adult intelligence quotient with the structure and function of the bilateral parieto-frontal networks, whereas the relationship between intelligence quotient and parieto-frontal network function has been found to be relatively weak in early childhood. Because both human intelligence and brain function undergo protracted development into adulthood, the purpose of the present study was to provide a better understanding of the development of the parieto-frontal network-intelligence quotient relationship.
\end{abstract}

MATERIALS AND METHODS: We performed independent component analysis of resting-state fMRI data of 84 children and 50 adolescents separately and then correlated full-scale intelligence quotient with the spatial maps of the bilateral parieto-frontal networks of each group.

RESULTS: In children, significant positive spatial-map versus intelligence quotient correlations were detected in the right angular gyrus and inferior frontal gyrus in the right parieto-frontal network, and no significant correlation was observed in the left parieto-frontal network. In adolescents, significant positive correlation was detected in the left inferior frontal gyrus in the left parieto-frontal network, and the correlations in the frontal pole in the 2 parieto-frontal networks were only marginally significant.

CONCLUSIONS: The present findings not only support the critical role of the parieto-frontal networks for intelligence but indicate that the relationship between intelligence quotient and the parieto-frontal network in the right hemisphere has been well established in late childhood, and that the relationship in the left hemisphere was also established in adolescence.

ABBREVIATIONS: $\quad I Q=$ intelligence quotient; FSIQ = full-scale intelligence quotient; PFN = parieto-frontal network; RS-fMRI = resting-state fMRI; TC-GICA = temporal-concatenation group independent component analysis; R.PFN = right PFN; L.PFN = left PFN; IFG = inferior frontal gyrus

$\mathbf{T}$ he brain basis of intelligence has been widely investigated for many years. ${ }^{1}$ A range of neuroimaging paradigms, including MR imaging, EEG, DTI, PET, and task-based fMRI, have been used to investigate the neural basis of intelligence quotient (IQ). ${ }^{2}$ A large percentage of these studies reported that individual differences in IQ are related to the structure or function of the parietofrontal regions in adults. ${ }^{3-6}$ For instance, Haier et $\mathrm{al}^{3}$ reported that more gray matter in the parieto-frontal regions was associated with

Received September 3, 2013; accepted after revision November 11.

From the Department of Biomedical Engineering, School of Computer and Information Technology, Beijing Jiaotong University, Beijing, China.

This work was supported by the National Natural Science Foundation of China (Grant Nos. 30800249, 61272356) and the Fundamental Research Funds for the Central Universities (Grant Nos. 2011JBM021, 2013JBZ003).

Please address correspondence to Lixia Tian, PhD, Department of Biomedical Engineering, School of Computer and Information Technology, Beijing Jiaotong University, Beijing 100044, China; e-mail: Ixtian@bjtu.edu.cn or tian.bme@gmail.com

- Indicates open access to non-subscribers at www.ajnr.org

Indicates article with supplemental on-line figures.

http://dx.doi.org/10.3174/ajnr.A3850 higher IQ on the basis of the MR imaging scans of adults. On the basis of MR imaging of young adults, Colom et $\mathrm{al}^{5,6}$ reported that the structural features of gray matter in the frontal lobes supported fluid, crystallized, and spatial intelligence. Jung and $\mathrm{Haier}^{2}$ reviewed 37 modern neuroimaging studies associated with intelligence and advanced the parieto-frontal integration theory, which indicated the importance of the parieto-frontal regions, along with some temporal and occipital regions, for intelligence.

Resting-state fMRI (RS-fMRI) is rapidly emerging as a powerful tool for in vivo mapping of neural circuitry in the human brain. Several recent studies investigated the neural basis of IQ in adults by use of RS-fMRI. ${ }^{7-10}$ Song et $\mathrm{al}^{7}$ analyzed the functional connectivities (FCs) associated with the bilateral dorsal lateral prefrontal cortex and reported that the FCs between parieto-frontal regions were particularly associated with intelligence. Van den Heuvel et $\mathrm{al}^{8}$ examined the RS-fMRI data from a complex network perspective and reported that the characteristic path length in parieto-frontal regions was longer in subjects with lower IQ. Wang et $\mathrm{al}^{9}$ reported the association between IQ and regional homogeneity of RS-fMRI signals in the parieto-frontal and other 
regions in adults. Cole et al ${ }^{10}$ reported a highly selective relationship between the global brain connectivity of left lateral prefrontal cortex and individual differences in fluid intelligence.

It should be noted that all these brain-intelligence relationship analyses were based on adults. Both mental abilities and brain function have been reported to undergo protracted development from early childhood into adulthood, ${ }^{11}$ and the higher-order association areas, including the parieto-frontal regions, have been reported to mature last. ${ }^{12,13}$ This indicates that the association between intelligence and function of the parieto-frontal regions might not have been established in children. Langeslag et $\mathrm{al}^{14}$ noticed this and analyzed the correlation between IQ and the FCs within the parieto-frontal networks (PFNs) across children 6-8 years old. On the basis of RS-fMRI, a significant functional connectivity-versus-IQ correlation was observed only in the right parieto-frontal network (R.PFN) at a relatively loose threshold $(P<$ .05 , false discovery rate-corrected for 10 comparisons), and this correlation in the left parieto-frontal network (L.PFN) was not significant $(P<.05$, false discovery rate-corrected for 3 comparisons) in their study. To date, the developmental route of the PFN-IQ relationship has not been well depicted, with a relatively weak relationship in early childhood, ${ }^{14}$ well-established relationship in adulthood, and unknown relationship in late childhood and adolescence.

To provide a better understanding of the development of PFN-IQ relationship, we analyzed the association between Wechsler full-scale IQ (FSIQ) and resting-state coactivation in the bilateral PFNs obtained by independent component analysis of RS-fMRI data. Independent component analysis is a powerful data-driven approach for finding independent patterns in multivariate data and one of the strongest statistical computational approaches in the detection of resting state networks. ${ }^{15,16}$ As compared with the extensively used seed-based functional connectivity analyses, independent component analysis needs no definition of seed regions and thus any possible bias caused by seedselection in seed-based functional connectivity analyses could be avoided by use of independent component analysis. We performed the study as follows: 1) on the basis of publicly released RS-fMRI data, temporal-concatenation group independent component analysis (TC-GICA) was performed to acquire the group independent component maps for the child group (84 subjects) and adolescent group (50 subjects) separately. Dual regression was then performed to find individual subject-level versions of the network spatial maps; 2) for each group, the components corresponding to the R.PFN and the L.PFN were identified by means of visual inspection, and voxelwise correlations with FSIQ were performed on the spatial maps of both the R.PFN and the L.PFN across subjects.

\section{MATERIALS AND METHODS \\ Subjects}

Data were selected from a large sample RS-fMRI dataset publicly released as "The ADHD-200 Sample" in the "1000 Functional Connectomes Project." 17 Subjects who satisfied the following 4 criteria were included in the present study: 1) typically developing subjects: the subjects had no lifetime history of head trauma with loss of consciousness and no history of neurologic illness or serious physical disease; 2) subjects enrolled at and data collected by
Beijing Normal University. As compared with those from other centers, the Beijing dataset included the largest sample size of typically developing subjects. We did not use data from multiple centers in consideration of avoiding any possible center-related variations, ${ }^{18}$ and the present sample size was relatively large (84 children and 50 adolescents); 3 ) head motion $<2.0 \mathrm{~mm}$ displacement in any of the $x, y$, or $z$ directions and $2.0^{\circ}$ of any angular motion throughout the resting-state scan; 4) subjects were righthanded, which was evaluated by the 10-item handedness inventory designed by The National Cooperative Research Team for Handedness of China. According to these 4 criteria, 134 subjects were included in this study, and were divided into 1 child group (84 subjects, 44 girls, 8.00-11.99 years old, with a mean age of $10.22 \pm 1.11$ years) and 1 adolescent group (50 subjects, 14 girls, $12.00-15.99$ years old, with a mean age of $13.47 \pm 0.81$ years).

\section{Dataset}

MR imaging data were obtained by use of a Trio 3T scanner (Siemens, Erlangen, Germany). Functional images were collected axially by use of an echo-planar imaging sequence sensitive to blood oxygen level-dependent contrast with the following parameters: 33 sections, $2000 / 30 \mathrm{~ms}$ (TR/TE), $90^{\circ}$ (flip angle), $220 \times 220 \mathrm{~mm}$ (FOV), $64 \times 64$ (resolution), 3.5/0.7 mm (thickness/gap). The resulting nominal voxel size was $3.44 \times 3.44 \times 4.50 \mathrm{~mm}$. The functional imaging lasted for 480 seconds. Whole-brain 3D T1weighted images were then obtained sagittally with the following parameters: 128 sections, 2530/3.39 ms (TR/TE), 1.33/0 mm (thickness/gap), $256 \times 256$ (resolution), $240 \times 240 \mathrm{~mm}$ (FOV), $7^{\circ}$ (flip angle). The resulting nominal voxel size was $1.07 \times 1.07 \times 1.33 \mathrm{~mm}$.

Subject FSIQ scores were assessed by use of the Wechsler Intelligence Scale for Chinese Children-Revised. Because the raw test scores were tailored to exact age, the FSIQ scores used in this study were independent of age. The mean FSIQ score of the 84 children was 119.4 (standard deviation $[\mathrm{SD}]=13.4$ ) and the range was $84-153$. The mean FSIQ score of the 50 adolescents was $115.2(\mathrm{SD}=12.7)$ and the range was $81-135$.

\section{RS-fMRI Data Preprocessing}

RS-fMRI data preprocessing was performed by use of FSL (http:// www.fmrib.ox.ac.uk/fsl). The following processing steps were applied to the RS-fMRI data of each subject: 1) removing the first 5 volumes; 2) correcting for head motion by use of the MCFLIRT tool in FSL; 3) removing the nonbrain tissues with the Brain Extraction Tool; 4) spatial smoothing by use of a Gaussian kernel of full width at half maximum $5 \mathrm{~mm}$; 5 ) high-pass temporal filtering to remove slow drift (cutoff frequency $=0.01 \mathrm{~Hz}$ ); 6) registering the subject's RS-fMRI data to his or her high-resolution structural image and then to Montreal Neurological Institute 152 standard space by use of the FLIRT and FNIRT tools in FSL and resampling the subject's registered RS-fMRI data to $2 \times 2 \times 2-\mathrm{mm}$ resolution. Because the effects associated with head motion and other noise could be ameliorated through the use of independent component analysis and dual regression, no denoising was performed.

\section{TC-GICA and Dual Regression}

TC-GICA was carried out on the datasets of the child group and adolescent group separately by use of MELODIC in FSL. Given 


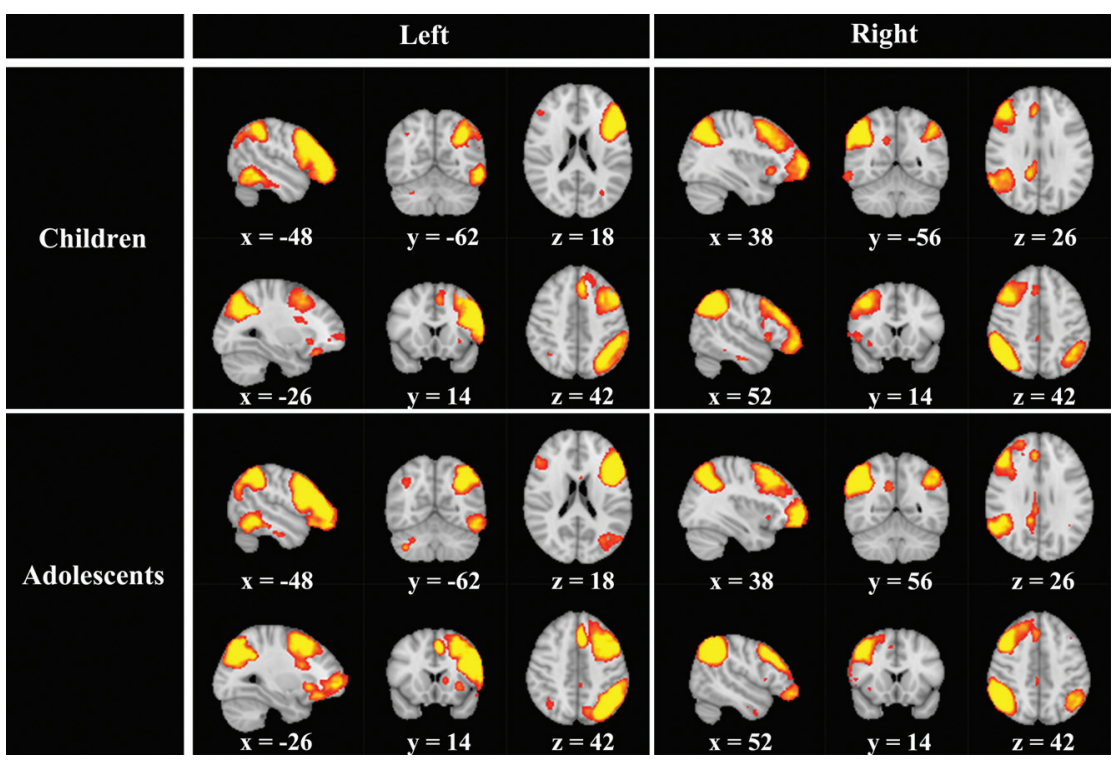

FIG 1. Spatial maps representing the parieto-frontal networks on the basis of temporal-concatenation group independent component analyses.

computational resource limitations, we performed a 2-stage TCGICA, as was used in the study by Biswal et al, ${ }^{18}$ as follows: 1 ) TC-GICA was performed 10 times, each with 20 randomly selected subjects, and the number of components was fixed at $20 .{ }^{16,18}$ 2) A second-stage independent component analysis was then carried out across the 10 runs to extract the 20 spatial maps consistently identified across the 10 runs. Dual regression was then applied to each subject's preprocessed RS-fMRI data to build individual-subject-level spatial maps. ${ }^{19-21}$ Specifically, for each subject (separately): 1) the formerly obtained 20 group-independent component analysis spatial maps (based on his or her own group dataset) were used as spatial regressors against the subject's 4D RS-fMRI data to estimate the network time-series of each component. Before being fed into the model, each component map was demeaned and normalized. 2) The resulting 20 timeseries were then used as temporal regressors against each subject's preprocessed 4D RS-fMRI data, resulting in 1 individual-subjectlevel spatial map for each component, reflecting how closely the functional data temporally resembled the component time-series at each voxel. Again, each time-series was demeaned and normalized before entering into the regression model.

\section{PFN-IQ Relationship Analyses}

PFN-IQ relationship analyses were performed on the spatial maps of the bilateral PFNs of the child group and adolescent group separately. To do this, we first identified the R.PFN and the L.PFN on the basis of visual inspection of the spatial maps generated by TC-GICA: the obviously right-lateralized network covering mainly the right parieto-frontal regions was identified as the R.PFN, and the network covering mainly the parieto-frontal regions in the left hemisphere was identified as the L.PFN. The spatial maps corresponding to the R.PFN/L.PFN were then collected across subjects into a $4 \mathrm{D}$ file (the fourth dimension was subject number). Five thousand permutations were finally performed within the corresponding PFN-mask obtained by thresholding the spatial map of the network on the basis of TC-GICA at
5 , to test for significant spatial map-versus-FSIQ correlations by use of the randomize command in FSL. Age, sex, and gray matter attenuation (acquired by voxel-based morphometry analysis of the 3D T1-weighted data, as a voxelwise covariate) were taken as covariates and removed. The results were thresholded at $P<.05$ (threshold-free cluster enhanced in FSL, family-wise error-corrected). ${ }^{22}$ To provide a comprehensive perspective about the PFN-IQ relationship at the 2 developmental stages, marginally significant correlations that survived a looser threshold of $P<.005$ (uncorrected) and cluster size $>200 \mathrm{~mm}^{3}$ (25 resampled voxels) were also reported.

To test whether the spatial map-versus-FSIQ correlations were specific to the bilateral PFNs, the medial visual network, the auditory-motor network, and the default mode network were selected as control RSNs, and 5000 permutations were also performed on the 3 control networks within the corresponding mask.

\section{RESULTS}

The R.PFN of the child group was similar to that of the adolescent group, and the L.PFNs of the 2 groups were also similar (Fig 1). The R.PFN and the L.PFN of each group appeared to be symmetrical. In each PFN, in addition to the ipsilateral parieto-frontal regions, small clusters were also observed in the presupplementary motor area and posterior cingulate cortex, the ipsilateral fusiform, as well as the contralateral parieto-frontal regions and cerebellum. Spatial maps of all 20 components of children and adolescents are shown in On-line Figs 1 and 2, respectively.

In the R.PFN of children, significant positive spatial mapversus-FSIQ correlations were found in the right angular gyrus and inferior frontal gyrus (IFG) $(P<.05$, threshold-free cluster enhanced, family-wise error-corrected), and positive correlations in left cerebellum and another right angular gyrus region were marginally significant (uncorrected $P<.005$ and cluster size $>200 \mathrm{~mm}^{3}$ ) (Fig $2 a-d$ and Table). No marginally significant negative correlation was found in the R.PFN of children. In the L.PFN of children, no marginally significant correlation (either positive or negative) was found.

In adolescents, significant positive correlations with the spatial map of the L.PFN were found in left IFG $(P<.05$, threshold-free cluster enhanced, family-wise error-corrected), and the positive correlation in the left frontal pole was marginally significant (uncorrected $P<.005$ and cluster size $>200 \mathrm{~mm}^{3}$ ) (Fig $2 e,-f$ and Table). Marginally significant positive correlation on the basis of the R.PFN was detected in the right frontal pole (Fig $2 g$ and Table). No marginally significant negative correlation was found in the 2 PFNs of adolescents.

No marginally significant spatial map-versus-FSIQ correlation (uncorrected $P<.005$ and cluster size $>200 \mathrm{~mm}^{3}$ ), either positive or negative, was detected in any of the 3 control RSNs of 

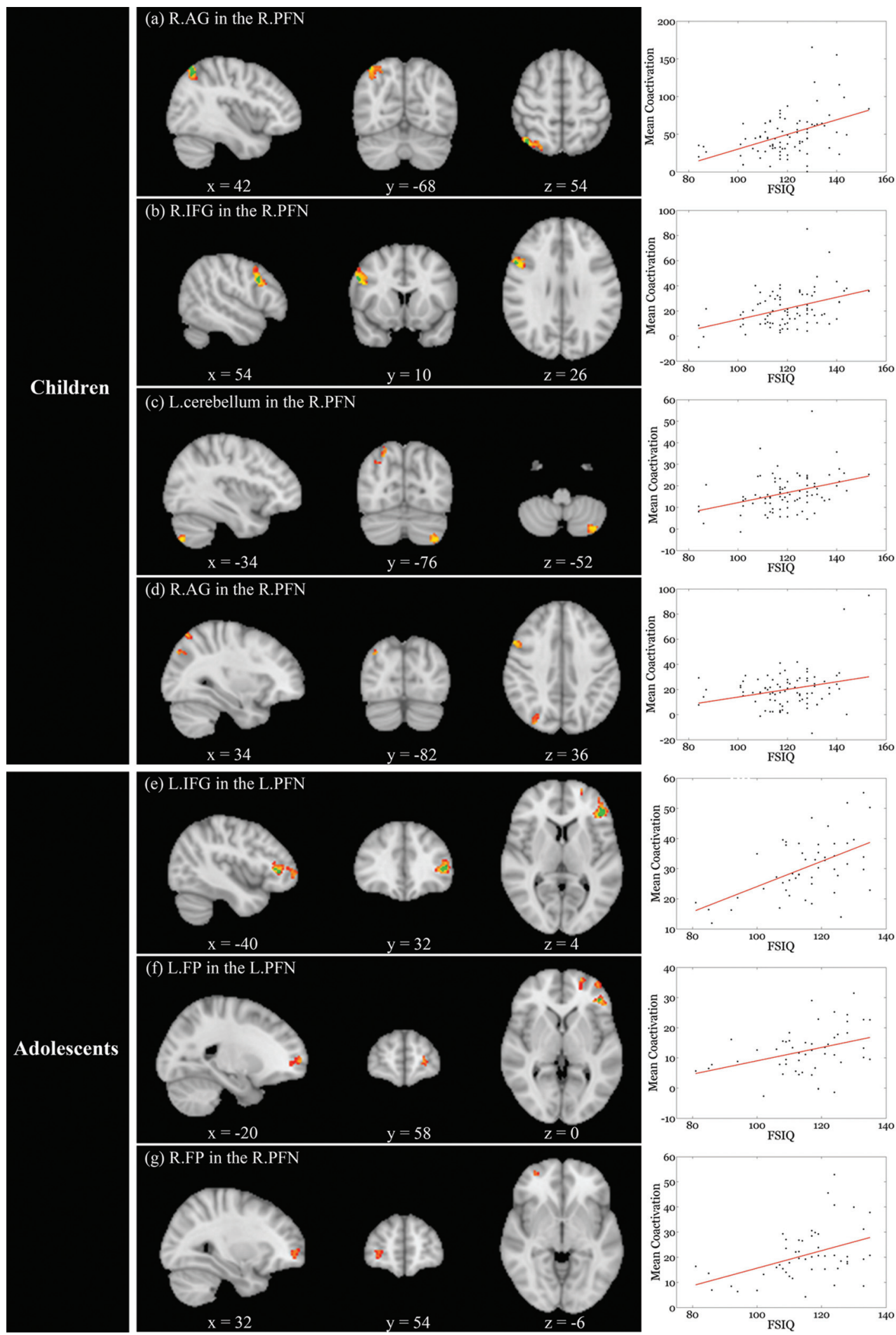

FIG 2. Positive correlations with full-scale intelligence quotient (FSIQ) across subjects. In the right parieto-frontal network (R.PFN) of children, significant correlations were observed in the right angular gyrus (AG) (a) and inferior frontal gyrus (IFG) (b), and marginally significant correlations were observed in the left cerebellum (c) and another right AG region (d). In adolescents, significant correlations were observed in the left IFG in the left PFN (L.PFN) (e), and marginally significant correlations were observed in the left frontal pole (FP) in the L.PFN (f) and right FP in the R.PFN (g). Green color indicates significant correlations and red-yellow color indicates marginally significant correlations. In the scatterplots, the $y$-coordinate indicates the mean coactivation of voxels exhibiting significant spatial map-versus-FSIQ correlations. 


\begin{tabular}{|c|c|c|c|c|}
\hline Region & Brodmann Area & Cluster Size, $\mathrm{mm}^{3}$ & MNI Coordinate (Peak) & $r$ Value (mean) \\
\hline \multicolumn{5}{|l|}{ Children: } \\
\hline \multicolumn{5}{|c|}{ Correlations with FSIQ in the R.PFN } \\
\hline R AG & 39 & $\begin{array}{c}184 \\
1656^{\mathrm{b}}\end{array}$ & $40,-66,56$ & 0.45 \\
\hline R IFG & 44 & $\begin{array}{c}128 \\
1616^{b}\end{array}$ & $52,12,28$ & 0.41 \\
\hline $\mathrm{L}_{\text {cerebellum }}{ }^{\mathrm{a}}$ & & $536^{\mathrm{b}}$ & $-36,-74,-50$ & 0.37 \\
\hline $\mathrm{RAG}^{\mathrm{a}}$ & 39 & $280^{b}$ & $32,-80,38$ & 0.27 \\
\hline \multicolumn{5}{|l|}{ Adolescents: } \\
\hline \multicolumn{5}{|c|}{ Correlations with FSIQ in the L.PFN } \\
\hline L IFG & 45 & $\begin{array}{c}256 \\
2416^{b}\end{array}$ & $-42,34,6$ & 0.54 \\
\hline $\begin{array}{l}\mathrm{LFP}^{\mathrm{a}} \\
\text { Correlations with FSI }\end{array}$ & 10 & $360^{\mathrm{b}}$ & $-22,60,2$ & 0.37 \\
\hline $\mathrm{RFP}^{\mathrm{a}}$ & 11 & $528^{b}$ & $30,56,-4$ & 0.43 \\
\hline
\end{tabular}

Note:-Threshold was $P<.05$ (threshold-free cluster enhanced, family-wise error-corrected)

${ }^{a}$ Marginally significant correlations (uncorrected $P<.005$ and cluster size $>200 \mathrm{~mm}^{3}$ ); ${ }^{b}$ represents the cluster size at this threshold. No marginally significant correlation was found in the L.PFN of children.

R indicates right; L, left; IFG, inferior frontal gyrus; AG, angular gyrus; FP, frontal pole; MNI, Montreal Neurological Institute.

any group (children and adolescents). This indicates that the spatial map-versus-FSIQ correlations were specific to the PFNs.

\section{DISCUSSION}

In this study, we obtained spatial maps by use of independent component analysis decomposition of RS-fMRI data of 84 older children and 50 adolescents and examined whether the PFN-IQ relationship had been established in each group. Only positive spatial map-versus-FSIQ correlations were detected in this study, and the correlations were specific to the PFNs. In children, significant/marginally significant positive correlations were found in the right angular gyrus and IFG and left cerebellum in the R.PFN (Fig $2 a-d$ and Table). In adolescents, significant positive correlations were found in the left IFG in the L.PFN, and the positive correlations in the frontal pole in the 2 PFNs were marginally significant (Fig $2 e-g$ and Table).

The importance of the PFN for IQ has been reported in many pioneering studies by use of diverse neuroimaging techniques. ${ }^{1-4,7,8,23}$ Specifically, more gray matter in the bilateral parieto-frontal regions has been reported to be associated with higher IQ. ${ }^{3,24}$ The fractional anisotropy within the bilateral frontal and occipito-parietal regions has been reported to be positively correlated with IQ. ${ }^{25}$ With task-based fMRI/PET, the parietofrontal regions have been reported to be active during working memory ${ }^{26}$ and a variety of reasoning tasks, ${ }^{23}$ both of which are important for intelligence. On the basis of graph-theoretical network analyses of EEG data, the degree centralities of parieto-frontal regions have been reported to be significantly correlated with intelligence. ${ }^{27}$ On the basis of RS-fMRI, IQ has been reported to be associated with functional connectivity between parieto-frontal regions, ${ }^{7}$ regional homogeneity of RS-fMRI signals in parietofrontal regions, ${ }^{9}$ global brain connectivity of the left lateral prefrontal cortex, ${ }^{10}$ and the normalized characteristic path length of parieto-frontal regions. ${ }^{8}$ In this study, significant/marginally significant spatial map-versus-FSIQ correlations were found in the right angular gyrus and IFG in the R.PFN of children (Fig $2 a, b$, and $d$ and Table), left IFG and frontal pole in the L.PFN, and right frontal pole in the R.PFN of adolescents (Fig $2 e-g$ and Table). The right IFG has been reported to play important roles in environ- ment monitoring, action planning, numerical ordering, and symbolic arithmetic. ${ }^{28,29}$ The left IFG has been repeatedly reported to be involved in phonological processing. ${ }^{30,31}$ Angular gyrus activations have been reported in such situations as spatial attention and orienting, language and semantic processing, and mathematical cognition. ${ }^{32,33}$ On the basis of meta-analysis, Bludau et $\mathrm{al}^{34}$ reported the involvement of the frontal pole in action selection, working memory, and perception. Each of the aforementioned functions of the 4 regions is critical for intelligence. Taken together, the present findings of significant/marginally significant spatial map-versus-FSIQ correlations in the PFNs (Fig 2 and Table) support the critical role of the PFNs for intelligence.

In this study, marginally significant spatial map-versus-IQ correlation was found in left cerebellum in the R.PFN of children (Fig $2 c$ and Table). In addition to its critical role in motor control, the cerebellum has been repeatedly reported to be involved in some cognitive functions. As for IQ, Frangou et $\mathrm{al}^{24}$ reported significant correlation between IQ and gray matter attenuation in cerebellum. Taki et $\mathrm{al}^{35}$ reported that the regional gray matter volume of cerebellum, together with that in bilateral prefrontal and temporo-parietal regions, was significantly positively correlated with FSIQ. The present finding of marginally significant correlation in the cerebellums of children is consistent with the 2 reports.

Only positive (and no negative) spatial map-versus-FSIQ correlations were found in this study. Formerly, decreased coactivation in certain resting-state networks has been linked to Alzheimer disease, ${ }^{36}$ obsessive-compulsive disorder, ${ }^{37}$ and cocaine addiction, ${ }^{38}$ and increased coactivation in certain resting-state networks has been linked to better performances in response inhibition ${ }^{21}$ and motor skill learning. ${ }^{39}$ In these studies, the extent of coactivation was deemed to represent the strength of functional interactions between the regions within the networks. If this were the case, the significant positive spatial map-versus-FSIQ correlations observed in this study may indicate that stronger functional interactions in the PFNs are advantageous for intelligence. According to the influential neural efficiency hypothesis of intelligence, ${ }^{40}$ brighter individuals would display lower brain activa- 
tion during cognitive task performance. Limited by datasets (no task data were available), the present results answered only the question about whether the PFN-IQ relationship exists at the 2 developmental stages. Further studies including both task and resting data may make it clear about how the PFN regions are involved in cognition.

The correlation in the R.PFN of children survived a familywise error-corrected threshold of $P<.05$, whereas the correlation in the R.PFN of adolescents survived only a looser threshold of $P<.005$ (uncorrected) and cluster size $>200 \mathrm{~mm}^{3}$. This does not mean that the R.PFN-IQ relationship become weaker with development. It should be noted that the sample size of children was 84 , and that of adolescents was 50 in this study. Because statistical power decreases with the decrease of sample size, the present finding of marginally significant correlations in adolescents may be interpreted, at least in part, by the smaller sample size of the group. Indeed, in this study, a correlation coefficient of 0.41 (right IFG in the R.PFN) in children corresponded to significant correlation, whereas a correlation of 0.43 (right frontal pole in the R.PFN) in adolescents only corresponded to marginally significant correlation (Table).

As has been mentioned, only a few studies have been performed specially for investigating the functional basis of IQ in children, and, to our knowledge, no study has been performed on the functional basis of IQ in adolescents, though numerous studies have been performed on that in adults. Langeslag et $\mathrm{al}^{14}$ noticed that both the brain and IQ underwent significant development before adulthood and proposed the importance of analyzing whether the PFN-IQ relationship had been established in children. They analyzed the correlation between IQ and the parietofrontal functional connectivity across children of 6-8 years old, on the basis of RS-fMRI. In their study, significant functional connectivity-versus-IQ correlation was observed only in the R.PFN at a relatively loose threshold $(P<.05$, false discovery rate-corrected for only 10 comparisons), and the correlation in the L.PFN was not significant $(P<.05$, false discovery rate-corrected for 3 comparisons). In the present study, the spatial mapversus-FSIQ correlations in the R.PFN of children (Fig $2 a,-b$ and Table) and L.PFN of adolescents (Fig $2 e$ and Table) were strong enough to survive an family-wise error-corrected threshold of $P<.05$ (threshold-free cluster enhanced, family-wise error-corrected for the number of voxels within the corresponding PFN mask). The present results bridge the gap between the findings that are based on adults and the results by Langeslag et $\mathrm{al}^{14}$ by indicating that during late childhood, the PFN-IQ relationship in the right hemisphere has been well established, and that relationship in the left hemisphere was also established during adolescence.

Both the study by Langeslag et $\mathrm{al}^{14}$ and the present study implicated a right-lateralized advantage in the development of PFN-IQ relationship. One speculative explanation for the findings is that the right hemisphere of a child's brain may develop earlier than its contralateral counterpart, and the left hemisphere may develop later to build a PFN-IQ relationship similar to that in adults. This speculation is consistent with former findings of rightward asymmetries of brain maturation in early developmental stages. For instance, the volume of the right hemisphere has been reported to be significantly larger than that of its left counterpart in children between $4-18$ years old. ${ }^{41} \mathrm{Zhu}$ et al ${ }^{42}$ reported that the FCs in the right hemisphere were stronger than those in the left hemisphere in children of $0-10$ years old. Zhou et $\mathrm{al}^{43}$ reported right-lateralized cortical thickness asymmetries in vision- and language-relevant areas in subjects of 5-14 years of age. Further studies are needed to provide a longitudinal view about the development of hemisphere-IQ relationship.

It should be noted that we investigated the PFN-IQ relationship based only on children of 8.00-11.99 years old and adolescents of 12.00-15.99 years old. These age ranges can provide only information about the extent of influences of resting-state coactivation in the PFN on IQ at the 2 developmental stages. To provide an overall view of the development of the PFN-IQ relationship, a study including samples covering a larger age range (eg, from 5 years old to adulthood) is needed. In addition, a larger sample size may enable a finer division of age ranges (eg, every 2 years rather than 4 years, as was used in the current study) and thus facilitate the acquisition of more detailed and precise understanding of the development of the PFN-IQ relationship. Furthermore, comparable sample size at each age range may provide an intuitive idea about the relative strength of the PFN-IQ relationship at different developmental stages.

\section{CONCLUSIONS}

We correlated FSIQ with the spatial maps of resting-state PFNs across 84 children and 50 adolescents to provide a better understanding of the development of the PFN-IQ relationship. The spatial map-versus-FSIQ correlations in the R.PFN of children and L.PFN of adolescents were strong enough to survive an family-wise error-corrected threshold of $P<.05$. The present findings not only supported the critical role of the PFNs for intelligence but bridged the gap between the results that were based on adults and those based on younger children (6-8 years old) by indicating that during late childhood, the PFN-IQ relationship in the right hemisphere has been well established, and that relationship in the left hemisphere was also established during adolescence. Further studies including a larger sample size and covering a larger age range are needed to better depict the developmental trajectory of the PFN-IQ relationship.

\section{ACKNOWLEDGMENTS}

The authors are grateful to the anonymous referees for their significant and constructive comments and suggestions, which greatly improved the study. The authors acknowledge the "1000 Functional Connectomes Project" for providing the dataset.

\section{REFERENCES}

1. Colom R, Jung RE, Haier RJ. Distributed brain sites for the G-factor of intelligence. NeuroImage 2006;31:1359-65

2. Jung RE, and Haier RJ. The Parieto-Frontal Integration Theory (PFIT) of intelligence: converging neuroimaging evidence. Behav Brain Sci 2007;30:135-54

3. Haier RJ, Jung RE, Yeo RA, et al. Structural brain variation and general intelligence. NeuroImage 2004;23:425-33

4. Jung RE, Haier RJ, Yeo RA, et al. Sex differences in $\mathrm{N}$-acetylaspartate correlates of general intelligence: a 1H-MRS study of normal human brain. NeuroImage 2005;26:965-72

5. Colom R, Haier RJ, Head K, et al. Gray matter correlates of fluid, 
crystallized, and spatial intelligence: testing the P-Fit model. Intelligence 2009;37:124-35

6. Colom R, Burgaleta M, Roman FJ, et al. Neuroanatomic overlap between intelligence and cognitive factors: morphometry methods provide support for the key role of the frontal lobes. NeuroImage 2013;72:143-52

7. Song M, Zhou Y, Li J, et al. Brain spontaneous functional connectivity and intelligence. NeuroImage 2008;41:1168-76

8. van den Heuvel MP, Stam CJ, Kahn RS, et al. Efficiency of functional brain networks and intellectual performance. $J$ Neurosci 2009;29:7619-24

9. Wang L, Song M, Jiang T, et al. Regional homogeneity of the restingstate brain activity correlates with individual intelligence. Neurosci Lett 2011;488:275-78

10. Cole MW, Yarkoni T, Repovs G, et al. Global connectivity of prefrontal cortex predicts cognitive control and intelligence. J Neurosci 2012;32:8988-99

11. Casey BJ, Tottenham N, Liston C, et al. Imaging the developing brain: what have we learned about cognitive development? Trends Cogn Sci 2005;9:104-10

12. Gogtay N, Giedd JN, Lusk L, et al. Dynamic mapping of human cortical development during childhood through early adulthood. Proc Natl Acad Sci U S A 2004;101:8174-79

13. Sowell ER, Thompson PM, Leonard CM, et al. Longitudinal mapping of cortical thickness and brain growth in normal children. J Neurosci 2004;24:8223-31

14. Langeslag SJ, Schmidt M, Ghassabian A, et al. Functional connectivity between parietal and frontal brain regions and intelligence in young children: the generation $\mathbf{R}$ study. Hum Brain Mapp 2013;34:3299-307

15. Beckmann CF, DeLuca M, Devlin JT, et al. Investigations into resting-state connectivity using independent component analysis. Philos Trans R Soc Lond B Biol Sci 2005;360:1001-13

16. Smith SM, Fox PT, Miller KL, et al. Correspondence of the brain's functional architecture during activation and rest. Proc Natl Acad Sci U S A 2009; 106:13040-45

17. ADHD-200 Sample. http://fcon_1000.projects.nitrc.org/indi/adhd200/. Accessed Jan 26, 2013

18. Biswal BB, Mennes M, Zuo XN, et al. Toward discovery science of human brain function. Proc Natl Acad Sci U S A 2010;107:4734-39

19. Filippini N, MacIntosh BJ, Hough MG, et al. Distinct patterns of brain activity in young carriers of the apoE-epsilon4 allele. Proc Natl Acad Sci U S A 2009;106:7209-14

20. Beckmann C, Mackay C, Filippini N, et al. Group comparison of resting-state fMRI data using multi-subject ICA and dual regression. In: 15th Annual Meeting of Organization for Hum Brain Mapp, poster 441 SU-AM. 2009

21. Tian L, Kong Y, Ren J, et al. Spatial vs temporal features in ICA of resting-state fMRI: a quantitative and qualitative investigation in the context of response inhibition. PLoS One 2013;8:e66572

22. Smith SM, Nichols TE. Threshold-free cluster enhancement: addressing problems of smoothing, threshold dependence and localisation in cluster inference. NeuroImage 2009;44:83-98

23. Fangmeier T, Knauff M, Ruff CC, et al. FMRI evidence for a threestage model of deductive reasoning. J Cogn Neurosci 2006;18:320-34

24. Frangou S, Chitins X, and Williams SC. Mapping IQ and gray matter density in healthy young people. NeuroImage 2004;23:800-05
25. Schmithorst VJ, Wilke M, Dardzinski BJ, et al. Cognitive functions correlate with white matter architecture in a normal pediatric population: a diffusion tensor MR imaging study. Hum Brain Mapp 2005;26:139-47

26. Gray WD, Schoelles MJ, Bringsjord SA, et al. SAGE: Five powerful ideas for studying and transforming the intelligence analyst's task environment. In: 47th Annual Conference of the Human Factors and Ergonomics Society 2003:1019-23

27. Langer N, Pedroni A, Gianotti LR, et al. Functional brain network efficiency predicts intelligence. Hum Brain Mapp 2012;33:1393-406

28. Knops A, Willmes K. Numerical ordering and symbolic arithmetic share frontal and parietal circuits in the right hemisphere. NeuroImage 2013;84C:786-95

29. Verbruggen F, Aron AR, Stevens MA, et al. Theta burst stimulation dissociates attention and action updating in human inferior frontal cortex. Proc Natl Acad Sci U S A 2010;107:13966-71

30. Bitan T, Cheon J, Lu D, et al. Developmental changes in activation and effective connectivity in phonological processing. NeuroImage 2007;38:564-75

31. MacSweeney M, Brammer MJ, Waters D, et al. Enhanced activation of the left inferior frontal gyrus in deaf and dyslexic adults during rhyming. Brain 2009;132:1928-40

32. Liang P, Wang Z, Yang Y, et al. Three subsystems of the inferior parietal cortex are differently affected in mild cognitive impairment. J Alzheimers Dis 2012;30:475-87

33. Esposito R, Mosca A, Pieramico V, et al. Characterization of resting state activity in MCI individuals. Peer J 2013;1:e135

34. Bludau S, Eickhoff SB, Mohlberg H, et al. Cytoarchitecture, probability maps and functions of the human frontal pole. NeuroImage 2014;93(Pt 2):260-75

35. Taki $Y$, Hashizume H, Sassa $Y$, et al. Correlation among body height, intelligence, and brain gray matter volume in healthy children. NeuroImage 2012;59:1023-27

36. Greicius MD, Srivastava G, Reiss AL, et al. Default-mode network activity distinguishes Alzheimer's disease from healthy aging: evidence from functional MRI. Proc Natl Acad Sci U S A 2004;101: 4637-42

37. Cheng $\mathrm{Y}, \mathrm{Xu}$ J, Nie B, et al. Abnormal resting-state activities and functional connectivities of the anterior and the posterior cortexes in medication-naive patients with obsessive-compulsive disorder. PLoS One 2013;8:e67478

38. Cisler JM, Elton A, Kennedy AP, et al. Altered functional connectivity of the insular cortex across prefrontal networks in cocaine addiction. Psychiatry Res 2013;213:39-46

39. Ma L, Narayana S, Robin DA, et al. Changes occur in resting state network of motor system during 4 weeks of motor skill learning. NeuroImage 2011;58:226-33

40. Neubauer AC, Fink A. Intelligence and neural efficiency. Neurosci Biobehav Rev 2009;33:1004-23

41. Giedd JN, Snell JW, Lange N, et al. Quantitative magnetic resonance imaging of human brain development: ages 4-18. Cereb Cortex 1996;6:551-60

42. Zhu C, Guo X, Jin Z, et al. Influences of brain development and ageing on cortical interactive networks. Clin Neurophysiol 2011;122: $278-83$

43. Zhou D, Lebel C, Evans A, et al. Cortical thickness asymmetry from childhood to older adulthood. NeuroImage 2013;83:66-74 